\title{
Far-infrared emission of massive stars ${ }^{\star}$
}

\author{
R. Siebenmorgen ${ }^{1}$, P. Scicluna ${ }^{2}$, and J. Krełowski ${ }^{3}$ \\ ${ }^{1}$ European Southern Observatory, Karl-Schwarzschild-Str. 2, 85748 Garching b. München, Germany \\ e-mail: Ralf.Siebenmorgen@eso.org \\ 2 Institue of Astronomy and Astrophyscis, Academia Sinica, 11F of AS/NTU Astronomy-Mathematics Building, No. 1, Sec. 4, \\ Roosevelt Rd, Taipei 10617, Taiwan \\ 3 Center for Astronomy, Nicolaus Copernicus University, Grudziądzka 5, Pl-87-100 Toruń, and Rzeszów University, al. T. Rejtana \\ 16c, 35-959 Rzeszów, Poland
}

Received 1 June 2018 / Accepted 4 September 2018

\begin{abstract}
We present results of the analysis of a sample of 22 stars of spectral types from O7 to B5 and luminosity classes I-V for which spectra from the Infrared Spectrograph (IRS) of Spitzer are available. The IRS spectra of these stars are examined for signs of excess infrared (IR) emission by comparison with stellar atmospheric spectra. We find that the spectra of half of the studied stars are dominated by excess emission in the far-IR, including all six super- and bright giants. In order to examine the origin of the far-IR excess, we supplement the Spitzer data with optical high-resolution echelle spectroscopy $\left(\lambda / \Delta \lambda \sim 10^{5}\right)$, near-IR high-contrast coronagraphic imaging taken with the SPHERE instrument at VLT with a spatial resolution of $0{ }^{\prime} 05$, and WISE and Herschel photometry. In the optical region, we detect various absorption and emission lines ( $\mathrm{H} \alpha, \mathrm{C}$ III, and N III) irrespective of the far-IR excess. Pfund $\alpha$ and Humphrey $\alpha$ lines are observed at the same time as the far-IR excess. These lines are stronger in stars with far-IR excess than in stars without excess. A scattered-light disk in the central $r \lesssim 2.5^{\prime \prime}$ region of the far-IR excess stars HD 149404, HD 151804, and HD 154368 can be excluded from $H$ band imaging down to a $1 \sigma$ contrast of $F(r) / F_{*} \sim 10^{-6}$. The far-IR excess is fit either by a free-free component from ionized gas as for the winds of hot stars or a large $(1 \mathrm{pc})$ circumstellar dust shell. The putative dust envelopes required to explain the excess have a visual extinction as low as a few hundred $\mu$-mag.
\end{abstract}

Key words. infrared: stars - dust, extinction - stars: early-type - infrared: ISM

\section{Introduction}

Thanks to the sensitivity of recent space missions, particularly the Spitzer and Herschel space telescope, infrared (IR) excess emission has been revealed for a large number of stars in a variety of environments. In low-mass stars, IR excess emission is commonly attributed to the presence of circumstellar dust, which absorbs stellar radiation and re-radiates it in the IR. This includes protoplanetary disks and AGB shells as well as debris disks. In more massive main sequence stars, excess emission is commonly attributed to thermal free-free emission in a hot, dense ionised wind (Hartmann \& Cassinelli 1977). This produces a continuum of emission from the IR to the radio with a distinctive power-law spectral index $f v \propto v^{0.7}$ (Barlow 1979). A particular case are Bestars emitting copious free-free radiation (Rivinius et al. 2013 for a recent review). However, in the IR it is difficult to distinguish free-free from optically thin dust emission. The presence or absence of dust could hold important information regarding sources of interstellar dust, particularly in high-redshift galaxies, and the formation and evolution of massive stars.

We present a sample of massive stars for which Spitzer/IRS (Houck et al. 2004) 5-35 $\mu \mathrm{m}$ spectra are available. These midto far-IR spectra ${ }^{1}$ are used to search for excess emission over

\footnotetext{
^ Optical high-resolution spectra are only available at the CDS via anonymous ftp to cdsarc.u-strasbg.fr (130.79.128.5) or via http://cdsarc.u-strasbg.fr/viz-bin/qcat?J/A+A/620/A32

1 We use terminology of near-IR including atmospheric windows in the JHK bands, mid-IR including LMN bands, and far-IR for longer wavelengths up to $200 \mu \mathrm{m}$.
}

the expected photospheric flux, which we seek to correctly attribute to wind and/or dust emission. In Sect. 2 we outline our sample selection and the data available for the stars. Section 3 discusses the modeling of the excesses as either wind or dust emission including the possible origins of the grains. Section 4 summarises our conclusions.

\section{Observations}

\subsection{Sample}

We select a sample of targets that forms a subsample of the Voshchinnikov \& Henning (2010) sample of 196 OB stars, which were originally selected for knowledge of the dust phase abundances of $\mathrm{O}, \mathrm{Mg}, \mathrm{Fe}$ and $\mathrm{Si}$ in the interstellar medium (ISM). The available data was extended in Voshchinnikov et al. (2012) by collating fractional polarisation measurements. In the optical region it is shown that the wavelength dependence of polarisation towards these stars follows a Serkowski curve (Bagnulo et al. 2017). The polarisation cannot therefore be due to scattering on dust or free electrons and must come from dichroic absorption by aligned dust particles. Further, it is assumed that the dichroism appears along the sightline towards the star in the diffuse ISM (Siebenmorgen et al. 2018), and ignoring any connection with the circumstellar environment of the stars. Voshchinnikov et al. (2012) study the role of the elements in the grain alignment that results in polarisation. Table 1 lists the relevant details of our sample, taken from the aforementioned papers, and references to the spectral types of the star. 
Table 1. Details of sample stars.

\begin{tabular}{|c|c|c|c|c|c|}
\hline Designation & RA & $\begin{array}{l}\text { Dec } \\
000\end{array}$ & SpT & $\begin{array}{c}V \\
(\mathrm{mag}) \\
\end{array}$ & $\begin{array}{c}E(B-V) \\
(\mathrm{mag}) \\
\end{array}$ \\
\hline HD 24912 & 03:58:57.90 & $+35^{\circ} 47^{\prime} 27.71^{\prime \prime}$ & O8IV & 4.06 & 0.35 \\
\hline HD 34816 & $05: 19: 34.52$ & $-13^{\circ} 10^{\prime} 36.44^{\prime \prime}$ & B0.5IV & 4.29 & 0.05 \\
\hline HD 36861 & $05: 35: 08.27$ & $+09^{\circ} 56^{\prime} 02.96^{\prime \prime}$ & O8III & 3.47 & 0.09 \\
\hline HD 38087 & 05:43:00.57 & $-02^{\circ} 18^{\prime} 45.38^{\prime \prime}$ & B3II & 8.29 & 0.31 \\
\hline HD 47839 & $06: 40: 58.66$ & $+09^{\circ} 53^{\prime} 44.72^{\prime \prime}$ & O7V & 4.64 & 0.07 \\
\hline HD 53367 & $07: 04: 25.53$ & $-10^{\circ} 27^{\prime} 15.74^{\prime \prime}$ & BoIV e & 6.96 & 0.74 \\
\hline HD 62542 & $07: 42: 37.22$ & $-42^{\circ} 13^{\prime} 47.84^{\prime \prime}$ & B3V & 8.03 & 0.36 \\
\hline HD 64760 & 07:53:18.16 & $-48^{\circ} 06^{\prime} 10.56^{\prime \prime}$ & B0.5III & 4.24 & 0.08 \\
\hline HD 65575 & $07: 56: 46.71$ & $-52^{\circ} 58^{\prime} 56.47^{\prime \prime}$ & B3IV & 3.43 & 0.05 \\
\hline HD 108639 & $12: 29: 09.51$ & $-60^{\circ} 48^{\prime} 17.55^{\prime \prime}$ & B1III & 8.57 & 0.35 \\
\hline HD 147933 & $16: 25: 35.10$ & $-23^{\circ} 26^{\prime} 48.70^{\prime \prime}$ & $\mathrm{B} 1.5 \mathrm{~V}$ & 5.05 & 0.47 \\
\hline HD 149404 & $16: 36: 22.56$ & $-42^{\circ} 51^{\prime} 31.90^{\prime \prime}$ & O8.5Ia & 5.52 & 0.62 \\
\hline HD 149757 & $16: 37: 09.54$ & $-10^{\circ} 34^{\prime} 01.53^{\prime \prime}$ & $09.2 \mathrm{~V}$ & 2.56 & 0.31 \\
\hline HD 151804 & $16: 51: 33.72$ & $-41^{\circ} 13^{\prime} 49.93^{\prime \prime}$ & O8Iab & 5.22 & 0.30 \\
\hline HD 154368 & 17:06:28.37 & $-35^{\circ} 27^{\prime} 03.77^{\prime \prime}$ & O9.5Ia & 6.13 & 0.76 \\
\hline HD 165024 & 18:06:37.87 & $-50^{\circ} 05^{\prime} 29.31^{\prime \prime}$ & B4II & 3.66 & 0.05 \\
\hline HD 188209 & 19:51:59.07 & $+47^{\circ} 01^{\prime} 38.42^{\prime \prime}$ & O9.5III & 5.63 & 0.15 \\
\hline HD 190918 & 20:05:57.32 & $+35^{\circ} 47^{\prime} 18.15^{\prime \prime}$ & O9.7Ia & 6.75 & 0.41 \\
\hline HD 192639 & $20: 14: 30.43$ & $+37^{\circ} 21^{\prime} 13.81^{\prime \prime}$ & O7.5Iab & 7.11 & 0.61 \\
\hline HD 206773 & $21: 42: 24.18$ & $+57^{\circ} 44^{\prime} 09.79^{\prime \prime}$ & B0V e & 6.87 & 0.45 \\
\hline HD 210839 & $22: 11: 30.58$ & $+59^{\circ} 24^{\prime} 52.15^{\prime \prime}$ & O6Ib & 5.05 & 0.57 \\
\hline HD 214680 & $22: 39: 15.68$ & $+39^{\circ} 03^{\prime} 00.97^{\prime \prime}$ & $09.5 \mathrm{~V}$ & 4.88 & 0.08 \\
\hline
\end{tabular}

Notes. We specify coordinates, spectral type, visual magnitude and extinction along the sightline, and reddening. Stars where we detect far-IR excess emission are marked in bold. HD 38087 is a binary, HD 53367 is a Herbig Be binary, HD 190918 is a WR+O star binary, HD 206773 is a Be star, HD 149404 is a multiple object, HD 214680 shows no sign of a binary in our two HR spectra despite that it is marked as a binary in SIMBAD.

\subsection{Archival data}

Our selection of stars is based on the availability of archival IRS (Houck et al. 2004) spectra, giving us a total of 22 stars. These data were complimented with optical (UBVRI) and near-IR (JHK) photometry obtained from Vizier/CDS and the 2MASS point-source catalogue (Cutri et al. 2003; Skrutskie et al. 2006). Where possible, further archival IR data were collected including those from the Infrared Array Camera (Fazio et al. 2004) of Spitzer, AKARI (Ishihara et al. 2010), the Infrared Astronomical Satellite (IRAS, Neugebauer et al. 1984; Joint Iras Science 1994), the Wide-Field Infrared Survey Explorer (WISE, Cutri et al. 2012) photometry, and Infrared Space Observatory (ISO) spectra from the Short Wavelength Spectrometer (SWS, Sloan et al. 2003). In most cases only upper limits are available. Unfortunately it has not been possible to locate higher quality data at wavelengths longer than $40 \mu \mathrm{m}$ from the Multi-Band Imaging Photometer (MIPS) of Spitzer. However, point-source photometry of the Photodetector Array Camera and Spectrometer (PACS) of Herschel (Marton et al. 2017) is available at 70, 100, and $160 \mu \mathrm{m}$ for HD 24912, HD 149404, HD 149757, HD 151804, and HD 210839.

\subsection{Detection of excess far-IR emission}

The photospheric flux of the sources is determined by taking a black-body spectrum appropriate to the spectral type of the star, giving a satisfactory fit to the 2MASS, WISE, and UBVRI photometry accounting for distance and foreground extinction. In the optical region the reddening correction is important. We take for each sightline the extinction curve and foreground extinction as given by Valencic et al. (2004); Fitzpatrick (2004), Fitzpatrick \& Massa (2007), and Gordon et al. (2009). We compare this estimate of the photospheric flux with stellar atmosphere models taken from the Castelli \& Kurucz (2004) atlas for the stellar type given in Table 1. The stellar atmosphere models are identical to black bodies at wavelengths $\geq 0.8 \mu \mathrm{m}$ and in the optical continuum when reddened by additional $0.1 \mathrm{mag}$. Given the precision of IR photometry we believe the uncertainties on photospheric parameters to be less than $10 \%$. Temperatures for the black body spectra were based on the Heap et al. (2006) calibration of O-star properties, and this was also used as a starting point for the luminosities. Figure 1 shows the IRS spectra and the contribution of the photospheres. The photospheric contribution thus determined was subtracted from the IRS spectrum to identify excess emission. The precision of our estimate of the emission by the photosphere is exemplified in Fig. 5. Apparently our single stellar temperature fits the optical/IR photometry well. The model provides a reasonable estimate of the far-IR emission of the photosphere, even for multiple-component systems such as HD 149404.

For 12 of the 22 sources, the fractional excess $\left(F_{\mathrm{IRS}}-F_{\mathrm{bb}}\right) / F_{\mathrm{bb}} \geq 0.1$ over a large portion of the IRS wavelength coverage, and for two stars, HD 149757 and HD 165024, the detected excess is marginal (Fig. 1). Ten of the stars are not considered host excess emission. Given the high signal-to-noise ratio $(\mathrm{S} / \mathrm{N})$ of our IRS spectra with absolute flux uncertainties of a few percent, this lower limit is somewhat conservative but allows for reasonable $(<10 \%)$ uncertainty in the photospheric parameters. The excess becomes clearly visible at wavelengths $\lambda \gtrsim 10 \mu \mathrm{m}$ and in Fig. 1 can be seen to be most pronounced at the longest wavelengths, such that we refer to it as far-IR excess. 


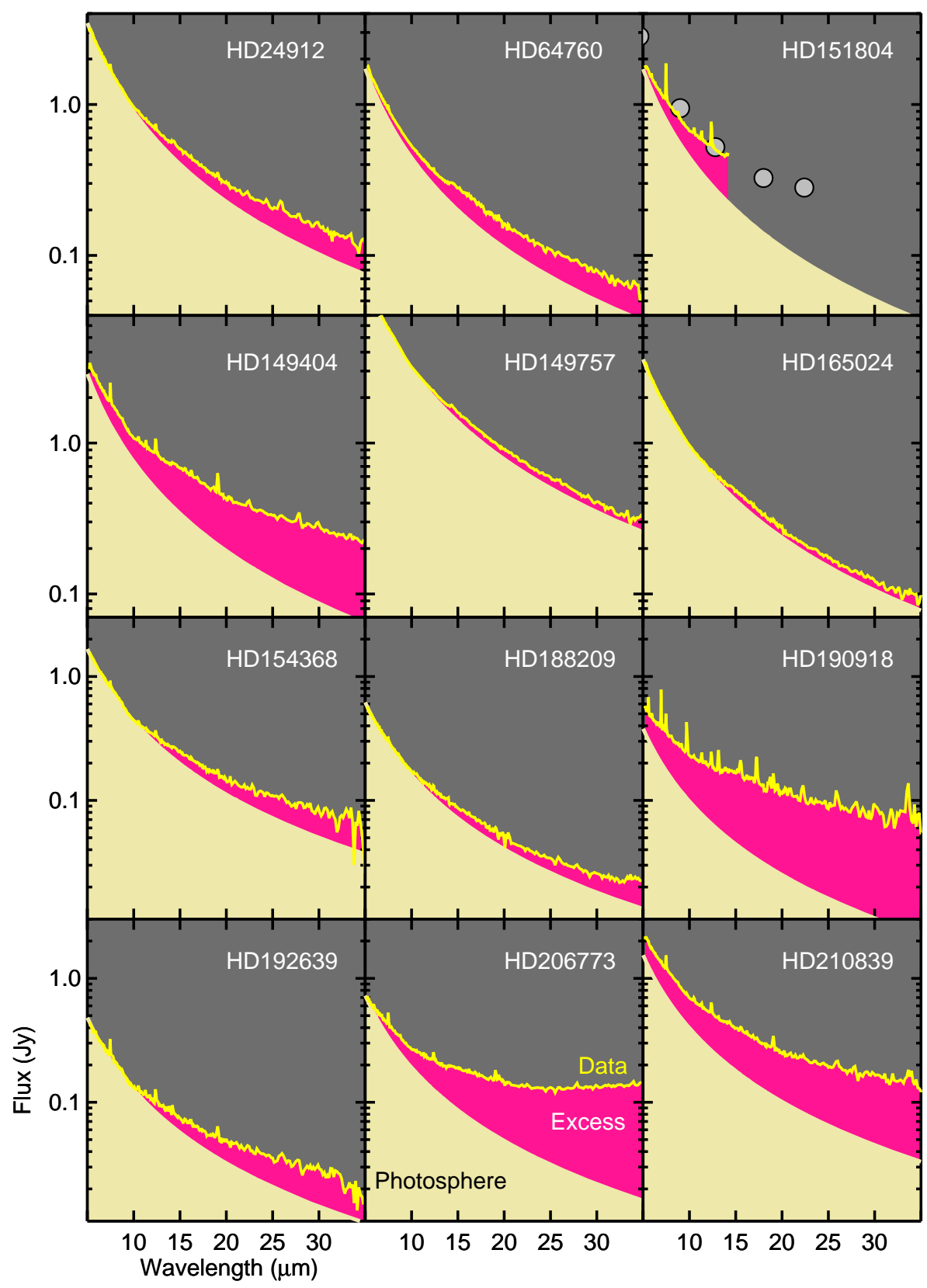

Fig. 1. Infrared (5-35 $\mu \mathrm{m})$ SEDs of sources. The yellow shaded region shows the photospheric contribution, while the yellow line shows the IRS spectrum. The magenta shaded region in between highlights the excess. Photometry for HD 151804 are shown as grey circles due to the restricted wavelength coverage of IRS.

\subsection{High-resolution spectroscopy}

For our sample, we collate high-resolution optical spectra observed over the past $25 \mathrm{yr}$. We have used ESO telescopes with instruments UVES/VLT offering a resolving power of $R=$ $\lambda / \Delta \lambda \sim 80000-110000$, HARPS $(R=115000)$ at the $3.6 \mathrm{~m}$, and FEROS $(R=48000)$ at the ESO/MPG $2.2 \mathrm{~m}$. We also used spectrographs at other observatories such as ELODIE $(R=$ $42000)$ at the $1.93 \mathrm{~m}$ Observatoire de Haute-Provence, GRAMS $(R=40000)$ at the $1 \mathrm{~m}$ of the Russian Special Astrophysical Observatory at Northern Caucasus, Sandiford $(R=64000)$ at the $2.1 \mathrm{~m}$ Otto Struve telescope, BOES $(R=30000-90000)$ at the $1.8 \mathrm{~m}$ Bohyunsan Optical Astronomy Observatory in Korea, and MAESTRO $(R=40000-120000)$ at the $2 \mathrm{~m}$ telescope of the Terskol Observatory at Northern Caucasus.

In Table 2 we list for each star the spectrograph used together with the observing date. We derive distance to the stars from Gaia DR2 parallaxes (Gaia Collaboration 2018), Ca II
(Megier et al. 2009), and from the spectral and luminosity class $(\mathrm{Sp} / \mathrm{L})$. We note that there is often a large scatter of up to a factor of two in the distance estimates (Krełowski 2018). We comment on the presence or absence of the $\mathrm{H} \alpha$ at $6563 \AA$, C III at $5596 \AA$, and N III at $4641 \AA$ lines and specify the strength of the Pfund $\alpha$ $(n=6 \rightarrow 5)$ near $7.5 \mu \mathrm{m}$ and Humphrey $\alpha(n=7 \rightarrow 6)$ transitions near $12.3 \mu \mathrm{m}$ (Kramida et al. 2018), as measured in the Spitzer/IRS spectrum. Pfund and Humphrey, C III and N III lines are detected in emission. Spectral classification of the stars is based on our optical HR spectra and reported in Table 1. Our classifications generally agree with those derived by Bowen et al. (2008), Jenkins (2009), Rauw et al. (2015); Skiff (2013); Sota et al. $(2011,2014)$.

Free-free radiation is emitted wherever there is ionised gas. The presence of hydrogen lines can also be a transient phenomenon and prototypical examples are Be-stars. The transient nature of the $\mathrm{H} \alpha$ emission in our sample is best demonstrated by HD 149757. The star is a rapid rotator and frequently 
Table 2. Spectral lines for stars with and without far-IR excess.

\begin{tabular}{|c|c|c|c|c|c|c|c|c|c|c|c|c|}
\hline \multirow[t]{2}{*}{ Star } & \multirow[t]{2}{*}{ Instr. $^{a}$} & \multirow[t]{2}{*}{ Date } & \multicolumn{3}{|c|}{ Distance } & \multirow{2}{*}{$\frac{\mathrm{H} \alpha}{6563 \AA}$} & \multirow{2}{*}{$\frac{\text { C III }}{5596 \AA}$} & \multirow{2}{*}{$\frac{\mathrm{N} \text { III }}{4641 \AA}$} & \multicolumn{2}{|l|}{$\operatorname{Pf} \alpha$} & \multicolumn{2}{|l|}{$\mathrm{Hu} \alpha$} \\
\hline & & & Paral. & $\begin{array}{l}\mathrm{Ca} I I \\
(\mathrm{pc})\end{array}$ & $\mathrm{Sp} / \mathrm{L}$ & & & & $\begin{array}{c}7.5 \mu \mathrm{m} \\
\left(10^{-17} \mathrm{~W} \mathrm{~m}^{-2}\right)\end{array}$ & $\begin{array}{l}\mathrm{EW} \\
(\AA)\end{array}$ & $\begin{array}{c}12.3 \mu \mathrm{m} \\
\left(10^{-17} \mathrm{~W} \mathrm{~m}^{-2}\right)\end{array}$ & $\begin{array}{l}\mathrm{EW} \\
(\AA)\end{array}$ \\
\hline \multicolumn{13}{|l|}{ Excess } \\
\hline HD 24912 & GRANS & 19960826 & 725 & 500 & 640 & No & Yes & Yes & $69 \pm 3.5$ & 67 & $10.5 \pm 0.8$ & 58 \\
\hline HD 64760 & UVES & 20090923 & 360 & 440 & 525 & Weak wings & No & No & $20 \pm 2.7$ & 37 & $3.9 \pm 0.7$ & 42 \\
\hline HD 149404 & UVES & 20090308 & 1315 & 1865 & 1240 & Yes double & Yes & Yes & $242 \pm 4.0$ & 224 & $41 \pm 1.0$ & 176 \\
\hline HD 149757 & HARPS & 20060310 & 112 & 230 & 240 & Weak $^{b}$ & No & No & $<12.8$ & - & $<2.9$ & - \\
\hline HD 151804 & FEROS & 20040502 & 1640 & 1680 & 1365 & Yes $+\mathrm{H} \beta$ & Yes & Yes & $307 \pm 3.3$ & 473 & $39 \pm 0.8$ & 254 \\
\hline HD 154368 & UVES & 20090306 & 1220 & 1290 & 1290 & Marginal & Yes & No & $39 \pm 2.8$ & 81 & $6.5 \pm 0.7$ & 72 \\
\hline HD 165024 & FEROS & 20040706 & 280 & 440 & 350 & No & No & No & $<11$ & - & $<2.6$ & - \\
\hline HD 188209 & BOES & 20050526 & 1490 & 1410 & 1270 & Yes & Yes & No & $9.7 \pm 1.9$ & 57 & $2.6 \pm 0.5$ & 84 \\
\hline HD $190918^{b}$ & BOES & 20070615 & 1960 & 2360 & 2290 & Yes & Yes & $?$ & $60 \pm 2.9$ & 274 & $9.4 \pm 0.8$ & 174 \\
\hline HD $192639^{b}$ & ELODIE & 20010811 & 2600 & 2080 & 2115 & Yes asym & Yes & Yes & $32 \pm 1.9$ & 221 & $3.0 \pm 0.5$ & 96 \\
\hline HD $206773^{b}$ & - & - & 960 & 550 & 595 & - & & - & $41 \pm 2.2$ & 154 & $6.8 \pm 0.6$ & 117 \\
\hline HD 210839 & MAESTRO & 20040828 & 620 & 1250 & 1190 & Yes & Yes & Yes & $134 \pm 3.2$ & 187 & $19 \pm 0.8$ & 129 \\
\hline \multicolumn{13}{|l|}{ No excess } \\
\hline HD 34816 & Sandiford & 19930210 & 270 & 410 & 380 & No & No & $?$ & $<7.2$ & - & $<1.6$ & - \\
\hline HD 36861 & UVES & 20040205 & 340 & 530 & 490 & No & Yes & Yes & $11.3 \pm 3.4$ & 16 & $<2.4$ & - \\
\hline HD 38087 & UVES & 20050321 & 340 & 470 & 490 & No & $?$ & No & $5.9 \pm 1.6$ & 121 & $<1.4$ & - \\
\hline $\mathrm{HD} 47839^{c}$ & GRAMS & 19960405 & 280 & 710 & 665 & No & Yes & Yes & $<6.3$ & - & $<1.7$ & - \\
\hline HD 53367 & UVES & 20091215 & - & 1000 & 780 & Yes & $?$ & Yes & $8.8 \pm 0.8$ & 39 & $1.1 \pm 0.2$ & 33 \\
\hline HD $62542^{b}$ & UVES & 20031113 & 390 & 450 & 530 & No & No & No & $0.14 \pm 0.04$ & 6 & $1.1 \pm 0.2$ & 33 \\
\hline HD $65575^{b}$ & - & - & 420 & 155 & 390 & - & - & - & $<10.0$ & - & $<2.3$ & - \\
\hline HD $108639^{b}$ & - & - & 1820 & 2380 & 2280 & - & - & - & $0.6 \pm 0.1$ & 16 & $0.19 \pm 0.03$ & 37 \\
\hline HD 147933 & HARPS & 20070330 & 145 & - & 100 & No & No & No & $<12.0$ & - & $<2.7$ & - \\
\hline HD 214680 & MAESTRO & 19660704 & 360 & 690 & 615 & No & No & No & $<5.9$ & - & $<1.5$ & - \\
\hline
\end{tabular}

Notes. We list for the optical high resolution spectra the name of instrument and date of the observations. Distance estimates are from Gaia, Ca II and from the spectral and luminosity class $(\mathrm{Sp} / \mathrm{L})$. The presence of the $\mathrm{H} \alpha, \mathrm{C} \mathrm{III}$, and N III lines is flagged. For the Pfund $\alpha(n=6 \rightarrow 5)$ and Humphrey $\alpha(n=7 \rightarrow 6)$ hydrogen transitions observed with IRS we give the continuum subtracted line fluxes and equivalent width. ${ }^{(a)}$ Optical high-resolution spectra of this instrument are made available at CDS. ${ }^{(b)}$ Parallax from Gaia (DR2) by the Gaia Collaboration (2018). ${ }^{(c)}$ Out of five spectra taken between 1993 and 2010 only the HARPS spectrum taken 2006-03-10 shows weak H $\alpha$ emission (Fig. 3).

classified as 09.5. We confirm the more recent O9.2IV classification by Sota et al. (2014), whereas Levenhagen \& Leister (2006) classified the star as Be-star (type B0Ve). Their classification is based on a spectrum taken in April 2000 at the ESO $1.2 \mathrm{~m}$. We have taken multiple high resolution spectra between May 1993 and March 2010 (Fig. 3). With the exception of a spectrum taken in 2006 where we observed weak $\mathrm{H} \alpha$ emission in the wings of the absorption line, $\mathrm{H} \alpha$ emission is not detected in any of these spectra of HD 149757. The Spitzer IRS spectrum taken in September 2008 displays Pfund $\alpha$ and Humphrey $\alpha$ in emission.

We present a second example underscoring the transient nature of the lines and show high-resolution FEROS and UVES spectra of HD 149404 in Fig. 2. The He I lines at 4471 and $5876 \AA$ observed with FEROS demonstrate that HD 149404 is a binary. One of the components shows the C IV $5812 \AA$ line and is therefore an $\mathrm{O}$ type star while the second does not show the C IV $5812 \AA$ line and is a B type star. The combined spectrum is apparently variable.

We detect $\mathrm{H} \alpha$ emission in $\sim 60 \%$ (7 out of 12) of the farIR excess stars and in only $14 \%$ ( 1 out of 7 ) of the non-far-IR excess stars. Therefore, we cannot firmly conclude from optical recombination lines whether a far-IR excess is present or not. Interestingly, the non-far-IR excess star HD 47839 displays C III and N III but no hydrogen lines despite it being classified as a Be-star.
Spitzer/IRS offers the unique capability of observing hydrogen lines simultaneously with the far-IR emission. This is of great advantage because of the transient nature of the lines. The strongest $\mathrm{H}$ recombination lines observed in the IRS domain are the Pfund $\alpha(n=6 \rightarrow 5)$ and Humphrey $\alpha(n=7 \rightarrow 6)$ transitions near 7.5 and $12.3 \mu \mathrm{m}$, respectively. Pf $\alpha$ lines are stronger than $\mathrm{Hu} \alpha$ lines. We find that there is no strong correlation between the detection of these lines and the presence of a farIR excess. Although from inspection of Table 2 a clear trend can be reported: the line intensities are typically an order of magnitude stronger in stars with far-IR excess than in stars without far-IR excess. The detection rate of the lines is $\sim 84 \%$ (10 out of 12) for IR-excess stars whereas $\mathrm{Pf} \alpha$ is detected only in $50 \%$ and $\mathrm{Hu} \alpha$ in $33 \%$ for stars without IR excess.

\subsection{High-contrast imaging}

The IRS spectra reveal a high detection rate of excess far-IR emission over the predicted photospheric component for 12 out of the 22 massive stars (55\%). We aim to resolve a dust component in a possible disk-like structure in scattered starlight using SPHERE, the extreme-adaptive-optics instrument at the VLT (Beuzit et al. 2008). The detection of scattered-light disks around massive stars would have presented interesting historical parallels to the detection of dusty disks around low-mass stars, such as $\beta$ Pic, which was first identified in the IR using IRAS before 

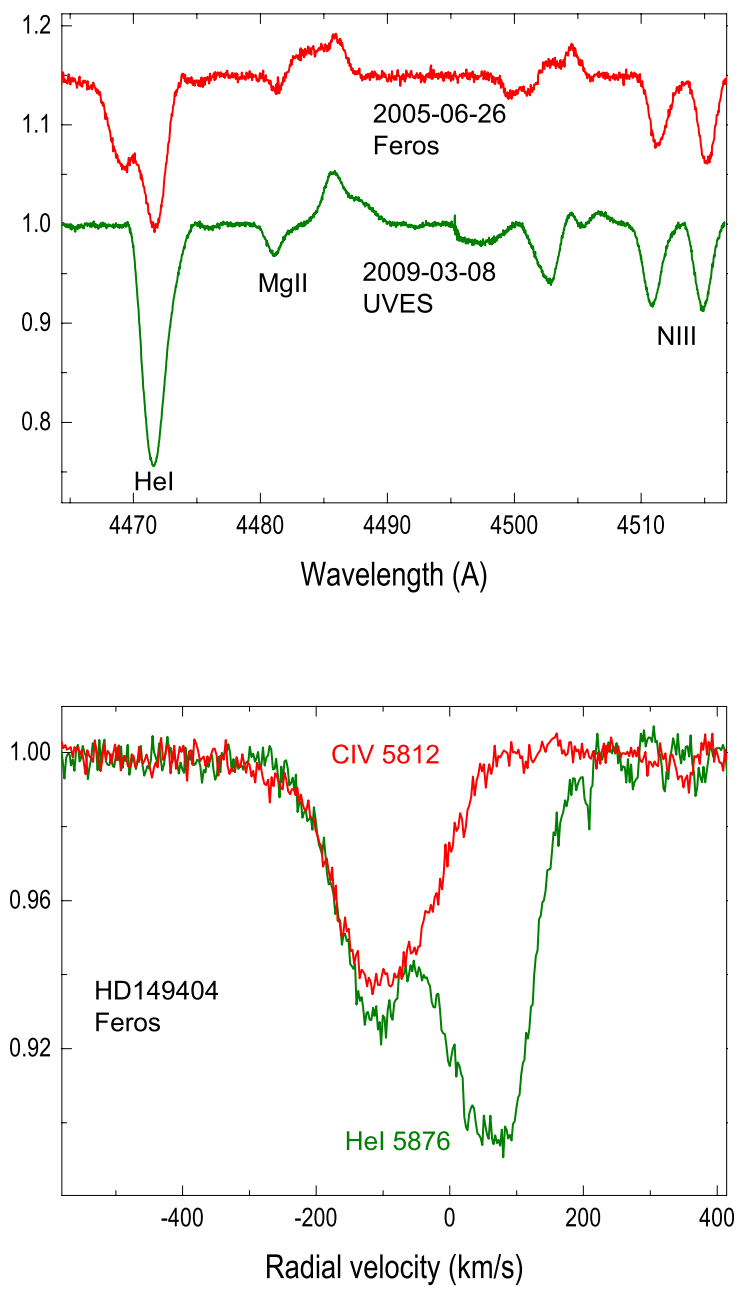

Fig. 2. High-resolution spectra (top panel) of HD 149404 taken with FEROS in 2005 (red) and UVES in 2009 (green). Bottom panel: velocity dispersion. One notices an additional component observed in the HeI $5876 \AA$ Aine, likely originated in a companion star, while in C IV such a second component is absent.

being confirmed by scattered-light imaging (Smith \& Terrile 1984).

We performed near-IR ( $H$ band) high-contrast coronagraphic imaging observations of three stars that show excess far-IR emission: HD 149404, HD 151804, and HD 154368, together with HD 147225 as a reference star. We used the IRDIS sub-instrument of SPHERE (Beuzit et al. 2008) on April 19 and June 19, 2015. The targets were observed for approximately $45 \mathrm{~min}$ in the broad $H$ band filter with a detector integration time of $0.84 \mathrm{~s}$ and a pixel scale of 12.27 mas. All exposures are flatfield corrected and background subtracted, their bad pixels are removed, and the detector dithering is corrected. Before adding the exposures, a subpixel shift algorithm is used to centre the images over time, and then the IRDIS detector channels are averaged. The procedure is performed on the science and reference star. The latter was used for point spread function (PSF) subtraction applying the algorithm described by Lafreniere et al. (2008). Finally, we compute the contrast given as the flux ratio $F(r) / F_{*}$ of science target and stellar halo as estimated from the PSF. The contrast is a function of distance $r$ (separation) from the star.

We do not observe any significant structures above the noise of the stellar halo that one could claim as scattered-light disk detection. A $1 \sigma$ noise limit of the contrast curve is computed.

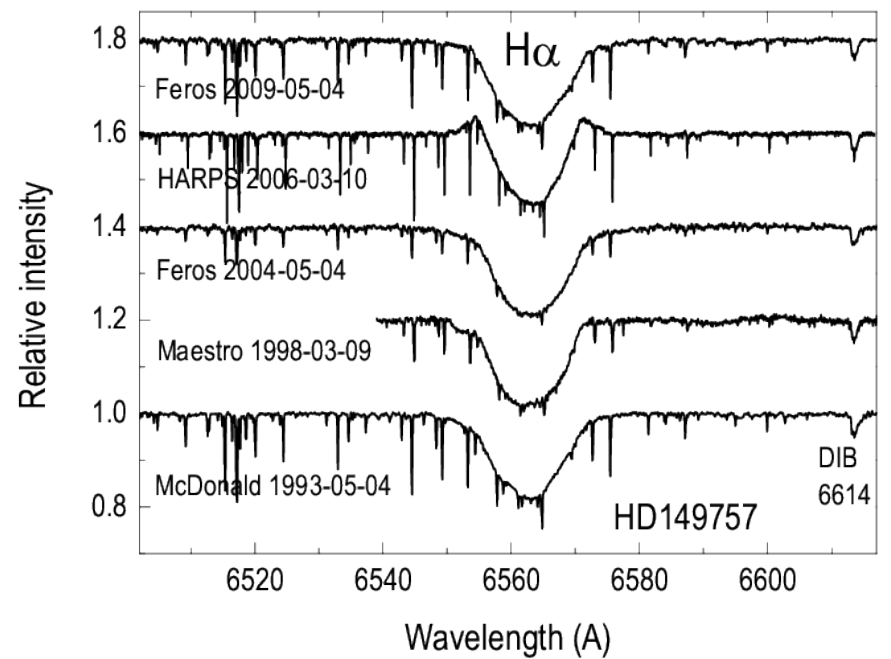

Fig. 3. High-resolution spectra of HD 149757 between observing epochs from 1993 to 2009 . $\mathrm{H} \alpha$ emission is detected only in the 2006 spectrum; we note the wings of the absorption profile.

It is given for each pixel separation as standard deviation in annuli with one-pixel width centred on the PSF core. Further details of the observing set-up and data reduction are given by Banas (2017) and Scicluna et al. (2017). The upper limits of the contrast curves of the three stars are displayed in Fig. 4. At $\sim 2^{\prime \prime}$ separation from the star, our data reaches a $1 \sigma$ contrast of $2 \times 10^{-7}$, demonstrating the unparalleled imaging contrast that can be achieved with SPHERE/IRDIS.

\section{Discussion}

After the detection of the far-IR excess in 12 out of $22 \mathrm{OB}$ stars, we explore the possible origin; we discuss the impact of background sources, the Be phenomenon, and emission from a faint dust halo around the sources. Spectral energy distribution (SED) models of the stars are best constrained when Spitzer spectra are available together with Herschel photometry. This is the case for five stars with far-IR excess that are listed in Table 3 (Col. 1) together with their stellar temperature (Col. 2), luminosity (Col. 3), and mass (Col. 4). To quantify the strength of the excess far-IR emission, we compute the ratio of the observed and photospheric luminosity in the IRS $5-35 \mu \mathrm{m}$ spectral range (Col. 5).

\subsection{Background source}

To identify possible contamination by dusty background sources, we fit a single-temperature modified black body, $f_{v} \propto v^{2} B_{v}(T)$, to the continuum excess using MPFIT (Markwardt 2009). The reduced $-\chi^{2}$ values are all $\gg 1$, indicating that a single black body is not adequate for fitting these spectra. Given the high temperatures of the best fits $(150-290 \mathrm{~K})$ it is also clear that a cool background source is not able to explain the excess.

\subsection{Be-stars}

Be-stars show a B-type stellar spectrum in combination with Balmer line emission, and an IR excess is often observed. The IR excess of Be stars is due to free-free emission from an ionized purely gaseous circumstellar disk and does not come from a wind or synchrotron component (Rivinius et al. 2013). For dense disks, the excess starts to dominate the photospheric emission at 
Table 3. Characteristics of far-IR excess sources with available Spitzer/IRS and Herschel photometry.

\begin{tabular}{|c|c|c|c|c|c|c|c|c|c|c|c|}
\hline 1 & 2 & 3 & 4 & 5 & 6 & 7 & 8 & 9 & 10 & 11 & 12 \\
\hline Source & \multicolumn{3}{|c|}{ Photosphere } & & & & \multicolumn{3}{|c|}{ Free-free emission } & \multicolumn{2}{|c|}{ Dust shell } \\
\hline & $\begin{array}{l}T_{\text {eff }} \\
(\mathrm{K})\end{array}$ & $\log \left(\frac{L_{*}}{L_{\odot}}\right)^{a}$ & $\begin{array}{c}M_{*} \\
\left(M_{\odot}\right)\end{array}$ & $\frac{L_{\mathrm{IRS}}}{L_{*}^{\prime}}$ & $\begin{array}{l}\dot{M} \\
(\log \end{array}$ & $\begin{array}{c}\dot{M}_{\mathrm{ff}} \\
\left.\frac{M_{\odot}}{\mathrm{vr}}\right)\end{array}$ & $\begin{array}{l}F^{\mathrm{ff}} \\
3.6\end{array}$ & $\begin{array}{l}F^{\text {wind }} \\
\text { m flux }\end{array}$ & $\begin{array}{c}F^{\mathrm{obs}} \\
\mu \mathrm{Jy})\end{array}$ & $\begin{array}{c}A_{\mathrm{V}, \mathrm{CS}}^{b} \\
(\mu \mathrm{mag})\end{array}$ & $\begin{array}{c}M_{\text {dust }}^{c} \\
\left(\mu M_{\odot}\right)\end{array}$ \\
\hline HD 24912 & 38000 & 5.3 & 31 & 1.2 & -6.2 & -5.5 & 258 & 30 & $<120^{d}$ & 95 & 62 \\
\hline HD 149404 & 33000 & 5.7 & 19 & 1.8 & - & - & 1533 & 300 & - & 140 & 92 \\
\hline HD 149757 & 33000 & 4.7 & 20 & 1.1 & -7.4 & -6.6 & 116 & 9 & - & 95 & 62 \\
\hline HD 151804 & 35800 & 5.8 & 22 & 1.4 & -5.0 & -4.6 & 987 & 300 & $400^{e}$ & 95 & 62 \\
\hline HD 210839 & 41000 & 6.0 & 21 & 2.1 & -4.7 & -4.7 & 602 & 600 & $430^{d}$ & 110 & 80 \\
\hline
\end{tabular}

Notes. ${ }^{(a)}$ Applying Sp/L distance as of Table 2. ${ }^{(b)}$ Visual extinction from inner to outer radius, and ${ }^{(c)}$ total dust mass. ${ }^{(d)}$ Puls et al. $(2006)$, ${ }^{(e)}$ Lamers \& Leitherer (1993).

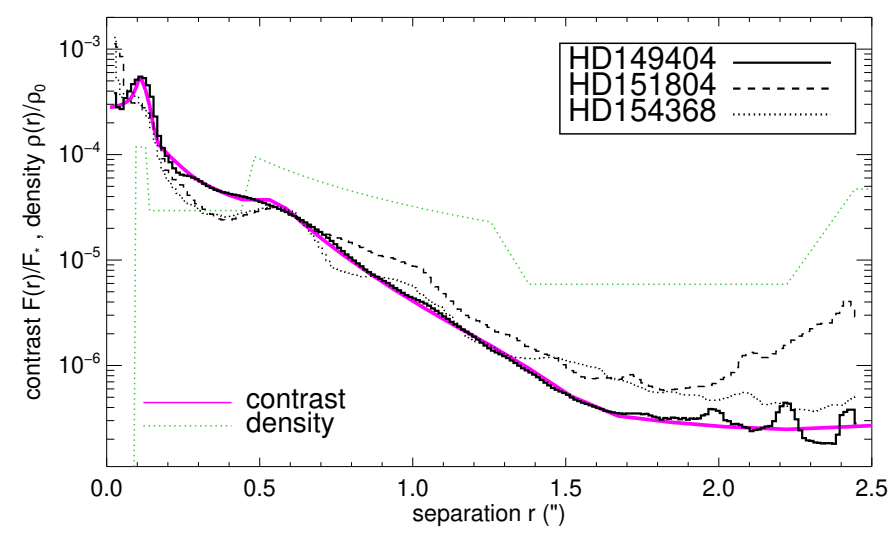

Fig. 4. SPHERE/IRDIS upper limits $(3 \sigma)$ of the contrast $F(r) / F_{*}$ (black) as a function of separation $r$ from the stars. A dust model fitting the upper limit of the contrast of HD 149404 (magenta line) is shown together with the applied dust density distribution $\propto \rho(r) / \rho_{0}$ (green dotted line).

near- to mid-IR wavelengths (Vieira et al. 2017). The slope of the IR excess depends on the density profile of the disk, and is close to a power-law $v^{\gamma}$ with $0.6 \lesssim \gamma \lesssim 2$ (Klement et al. 2017). The Be phenomenon may be a period in the life of a "normal" B star (Galazutdinov \& Krełowski 2006).

We searched various catalogues and publications listing Be-stars (Fabregat et al. 1996; Rivinius et al. 2006; Wisniewski et al. 2007; Catanzaro 2013; Draper et al. 2014; Chojnowski et al. 2015; Lin et al. 2015) and find three stars in our sample that were classified as Be-type stars. There is HD 47839 that does not display a far-IR excess, HD 149757 ( $\zeta$ Oph) that occasionally shows Be-type outbursts (Vogt \& Penrod 1983; Kambe et al. 1997, Fig. 3), and HD 206773 that has a strong far-IR excess with a rising wavelength dependency at $\gtrsim 25 \mu \mathrm{m}$ (Fig. 1). The occurrence of three likely Be-stars in our sample of 22 stars is in line with the estimate that about $17 \%$ of B-type stars are Be-stars (Zorec \& Briot 1997). However, we detect a farIR excess in 12 out of 22 sources at a much higher detection rate. Fully ionized optically thick disk models of Be-stars are presented by Carciofi \& Bjorkman (2006). They predict excess emission for disks inclinations $\leq 60^{\circ}$ emerging at short wavelengths $(\lesssim 1 \mu \mathrm{m})$ and for edge-on view $\left(90^{\circ}\right)$ in the mid-IR at $\sim 10 \mu \mathrm{m}$. The excess that we are observing develops in the far-IR at wavelengths $\gtrsim 10 \mu \mathrm{m}$ (Fig. 1). It is unlikely that all our far-IR excess stars are yet unclassified Be-stars with their disks viewed nearly edge-on. Therefore, the Be-star phenomenon is unlikely to be the dominant origin of the far-IR excess.

\subsection{Wind models}

It has been shown (Barlow 1979) that a dense ionised medium generates free-free emission at wavelengths from radio to IR. A simple $F_{\mathrm{ff}} \propto v^{0.7}$ dependence has been shown to fit well over six orders of magnitude in frequency from the IR to the radio (Klement et al. 2017). It is apparent for the strongest radioemitters (e.g. HD 210839) that an IR free-free component is present. In order to investigate this effect we located relevant radio data for our targets where available. For those stars with no radio data, it is possible to estimate a theoretical mass-loss rate $\dot{M}$ (Vink et al. 2001), as a function of the mass, luminosity, temperature and metallicity of the source ${ }^{2}$. As a result, it is necessary to determine masses for our sample of stars. To do so, we compare the luminosity and temperature to the Geneva evolutionary tracks (Ekström et al. 2012) for solar metallicity to find a best fitting mass. Multiple systems should be excluded from such an analysis. The mass-loss rates thus derived are then used to predict a $3.6 \mathrm{~cm}$ flux for the wind $F^{\text {wind }}$. The flux estimate is based on

$\dot{M}=5.32 \times 10^{-4} \frac{\mu D^{\frac{3}{4}} v_{\infty}}{\sqrt{\tilde{g} v}}\left(F_{v}^{\mathrm{wind}}\right)^{\frac{3}{4}}\left(\frac{M_{\odot}}{\mathrm{yr}}\right)$,

where $D$ is the distance, $v_{\infty}$ the wind thermal velocity, and $\tilde{g}$ is the Gaunt factor assuming an effective wind temperature $T_{\text {wind }} \sim 0.6 T_{*}$ (Carciofi \& Bjorkman 2006). In the model by Vink et al. (2001) it is assumed that the wind is composed entirely of ionised hydrogen, and so the mean ionic weight $\mu=0.5$ in units of proton mass. The derived mass-loss rates are given in Table 3 (Col. 6) together with the $3.6 \mathrm{~cm}$ flux $F_{\text {wind }}$ (Col. 8). We extrapolate the fit to the IR data by the free-free component to a $3.6 \mathrm{~cm}$ flux $F_{\mathrm{ff}}(\mathrm{Col} .7)$, which using Eq. (1) gives a mass-loss rate $\dot{M}_{\mathrm{ff}}$ specified in Col. 7 . Whenever available we give the observed $3.6 \mathrm{~cm}$ flux $F_{\text {obs }}$ in Col. 9 .

In Fig. 5 (left panels) the free-free component $F^{\mathrm{ff}}$ is shown using a blue dotted line, and the total flux $F_{*}+F_{\mathrm{ff}}$ using a green full line. We find that the free-free component can always be adjusted so that IR data are matched. The SEDs of HD 149757 and HD 210839 are well fit when assuming that the free-free flux is given by the wind models $\left(F_{\mathrm{ff}}=F_{\text {wind }}\right)$ while such a model underestimates IR data of the other sources. However, the

2 Using a routine from http://star.arm.ac.uk/ jsv/Mdot.pro. 


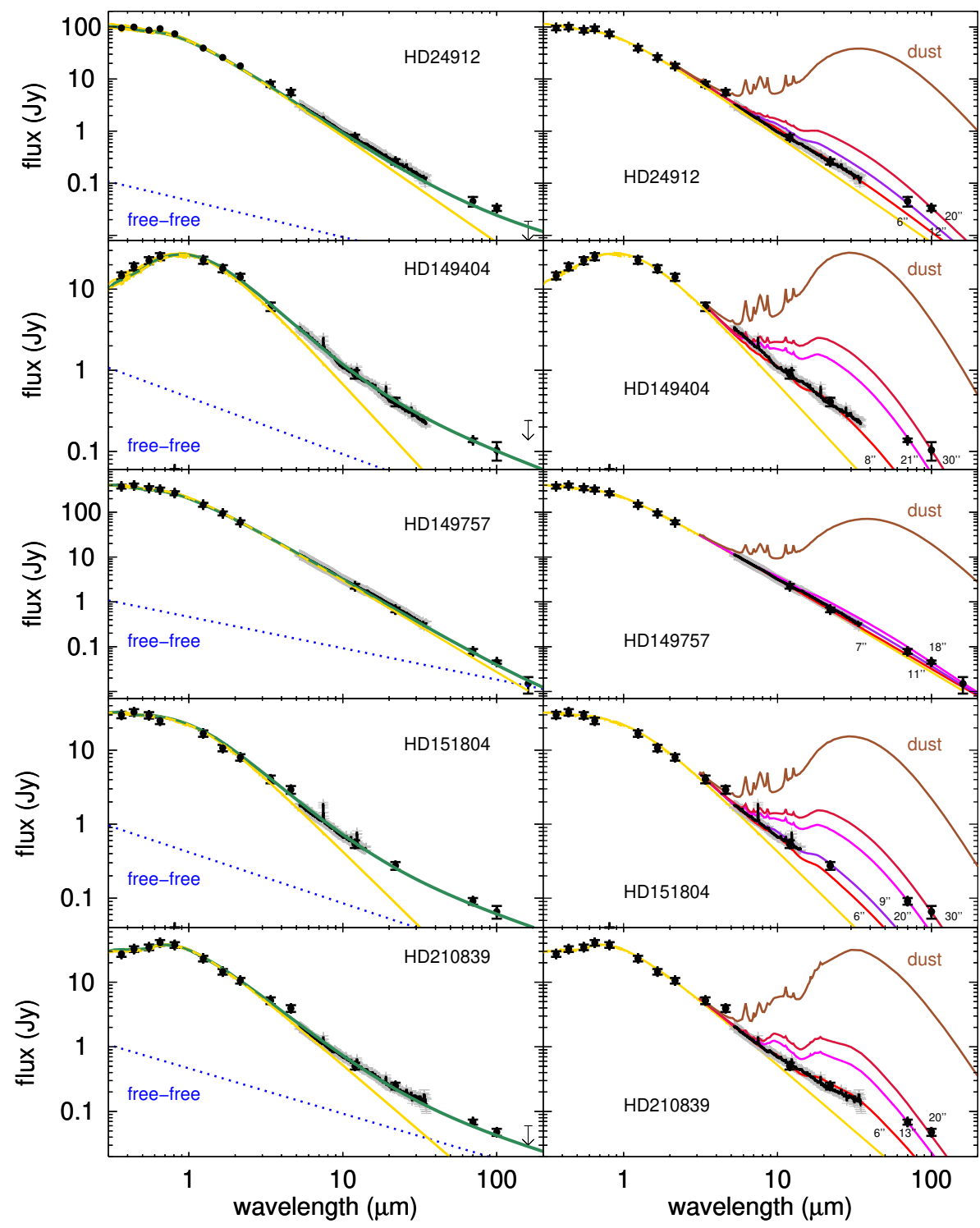

Fig. 5. SED of massive stars. The total emission is fit by adding to the photosphere (yellow) either free-free (left blue dotted lines) or dust emission in apertures as labelled (right red-brown lines). Data (black) are from optical catalogues as available at VizieR/CDS, 2MASS, WISE, Spitzer/IRS, and Herschel/PACS with $1 \sigma$ error bars and $3 \sigma$ upper limits. required $3.6 \mathrm{~cm}$ free-free flux is much stronger (factor $\sim 2$ ) than the observed fluxes of HD 24912 and HD 210839 by Puls et al. (2006) and HD 151804 by Lamers \& Leitherer (1993).

\subsection{Circumstellar dust models}

As a single dust temperature cannot reproduce the observed emission, we require a distribution of dust near the source with a range of temperatures and masses: a circumstellar envelope. The data for each source were modelled assuming spherical symmetry using a code described in Krügel (2008) with dust heated by a central star. The code solves the radiative transfer equation by ray tracing, with the source placed at the centre of the cloud. We solve the radiative transfer equation

$I_{v}(\tau)=I_{v}(0) e^{-\tau}+\int_{0}^{\tau} S(x) e^{x-\tau} \mathrm{d} x$,

with the source function

$S_{v}=\frac{K_{v}^{\mathrm{abs}} \int P(T) B_{v}(T) \mathrm{d} T+\left(1-g_{v}\right) K_{v}^{\mathrm{sca}} J_{v}}{K_{v}^{\mathrm{ext}}}$,

where $I_{v}$ is the intensity, $B_{v}(T)$ is the Planck function, $P(T)$ is the temperature distribution for stochastically heated grains
(Siebenmorgen et al. 1992), $K^{\text {abs }}$ is the absorption, $K^{\text {sca }}$ the scattering, $K^{\text {ext }}$ the extinction cross-sections, $g$ is the scattering anisotropy parameter, and $\tau$ is the optical depth. By multiplying the scattering cross-section by $1-g$ we are able to account for anisotropic scattering by effectively dividing the scattering into a purely isotropic component and a forward scattered component (Scicluna \& Siebenmorgen 2015). The boundary conditions are provided by the external radiation field and the stellar flux. The input stellar spectrum is treated as a black body of given temperature and integrated luminosity truncated at Ly $\alpha$, which is then processed by the dust distribution. It is not necessary to treat an interstellar radiation field as the luminosity of the stars is so great that they dominate the local radiation field (Mathis et al. 1983). Krügel (2008) gives the interstellar radiation field a mean value of $0.04 \mathrm{erg} \mathrm{s}^{-1} \mathrm{~cm}^{-2}$, while for a star of luminosity $10^{4} L_{\odot}$ the radiation field at $1 \mathrm{pc}$ is $0.32 \mathrm{erg} \mathrm{s}^{-1} \mathrm{~cm}^{-2}$, an order of magnitude larger. The orbital separations of the multiple stars included in the sample are small compared to the expected dust inner radius, so we model them as a single point source.

The dust model consists of sub-micron sized grains of amorphous carbon and/or silicates, as well as stochastically heated particles in the form of PAHs and nanometre-scale graphite 
and silicate particles (Siebenmorgen et al. 2014). We apply dust parameters for single-cloud sightlines given by Siebenmorgen et al. (2018). The radiative transfer is solved iteratively, and dust emission and temperatures are solved self-consistently. The geometry of the envelopes is characterised by an inner and outer dust radius, and a dust density distribution $\rho(r)$. The model allows the computation of SEDs within apertures of the circumstellar envelope of different angular sizes.

We demonstrate the models using the strong far-IR excess source HD 149404 for which a SPHERE contrast curve and Herschel photometry are available. Its photosphere is approximated by a single temperature of $33000 \mathrm{~K}$, which is reddened by $A_{\mathrm{V}}=2.2 \mathrm{mag}$, and a Fitzpatrick (2004) and Gordon et al. (2009) extinction curve with $R_{\mathrm{V}}=3.65$. As shown in Fig. 5, the photosphere model fits the optical (UBVR), 2MASS, and WISE photometry despite the variability of this multi-component system (Fig. 2). The lower panel of Fig. 2 shows an additional component in the HeI $5876 \AA$ line representing the fainter O-type companion star, while such a second component is absent in the C IV $5812 \AA$ line. WISE photometry at 12 and $22 \mu \mathrm{m}$ is consistent with the Spitzer IRS spectrum.

We take the SPHERE contrast curve to constrain the innermost $0.05^{\prime \prime}-2.5^{\prime \prime}$ region of the dust shell (Fig. 4). The IR data are fit assuming a constant dust density in a shallow dust envelope in the range $0.011<r \leq 1$ (pc) of $A_{\mathrm{V}}=300 \mu \mathrm{mag}$. We also compute a second model in which the dust content in the inner region $(\leq 2275 \mathrm{AU})$ is increased by multi-component power laws until the $3 \sigma$ upper limit of the contrast curve is matched. This gives an upper limit of the total amount of warm $(\gtrsim 250 \mathrm{~K})$ dust of $\lesssim 10^{-10} M_{\odot}$. The density distribution in the inner region is shown as green and the contrast curve of that model is shown with a magenta line in Fig. 4. The low amount of warm dust does not contribute to the SED in the IRS spectral range. For HD 24912, HD 149757, and HD 151804, we keep the same structure of the circumstellar dust shell and set $\rho(r)=\rho_{0}$ to be constant for $0.011<r \leq 1$ (pc) and otherwise there is no dust. For HD 210839, our highest-luminosity far-IR excess star, we increased the inner radius to $0.026 \mathrm{pc}$ so that the model does not show the $\mathrm{SiO}$ dust bands. At the inner radius, the dust temperature is about $300 \mathrm{~K}$. Nano-sized particles are photo-dissociated up to distances of $\sim 6 \times 10^{16} \mathrm{~cm}$ from the star. The models are consistent with the non-detection of PAH features or oxygenrich dust bands (silicates) in the IRS spectra. The dust emission spectrum is insensitive to the adopted effective temperature of the OB stars. The computed dust emission spectra are identical when stars of the same luminosity and of spectral shapes ranging between 30000 and $40000 \mathrm{~K}$ are considered. Therefore, we only allow $\rho_{0}$ to vary as a free parameter when fitting the far-IR data. The corresponding extinction $A_{\mathrm{V}, \mathrm{CS}}$ and dust mass $M_{\mathrm{D}}$ are listed in Table 3. Spectral energy distribution models are shown for different observing apertures of the circumstellar dust shells in the right panels of Fig. 5. A similar quality fit is achieved for the SEDs using either the free-free or dust model, and so a preference for one or the other model was not decided upon. At the spatial resolution of ALMA the extended dust halo model predicts submillimetre fluxes that are orders of magnitude fainter than the point-like emission that is estimated by the free-free model. Therefore, the nature of the far-IR excess can be tested by future ALMA observations in the submillimetre continuum. A similar argument holds for observations in the radio, however there is the underlying assumption that one can extrapolate the power law of the free-free component over many orders of magnitude in frequency.

\subsection{Dust formation}

The observed far-IR excess may be due to free-free or dust emission, and for the latter, one wonders how grains may sustain the harsh environment of the OB stars. For A stars, the blow-out radius or the dust grain radius below which all grains experience greater radiation pressure forces than gravitational is several micrometres (Beust 2010). The blow-out radius is proportional to $L_{*} / M_{*}$ implying that it is between 1 and 2 orders of magnitude larger for OB stars. This means that sub-micrometre sized grains should be rapidly removed and only much larger particles would remain in the stellar environment. Another important effect to consider is Poynting-Robertson drag, which causes dust grains to lose angular momentum and spiral toward the star (Draine 2011). Once again, this effect is stronger for smaller grains around more luminous stars and would cause grains between 0.1 and $1 \mathrm{~mm}$ to spiral in from the inner radius of our models on timescales of $10^{6}-10^{7} \mathrm{yr}$. Therefore, this dust population is not long-lived and must be replenished from some source. A replenishment similar to collisional cascades observed by debris-disk emission (Su et al. 2013) is unlikely because of the non-detection of a scattered-light disk in the high-contrast imaging observations with SPHERE of at least three of our far-IR excess stars.

One possible cause for stars to have both dust and wind emission is that dust formation takes place in the winds. This is most likely occurring in multiple-systems where the wind collisional region between the two components is likely to reach extremely high densities, allowing the condensation of dust particles, as in, for example, the WC7+O5 binary WR140 (Williams 2008). From our data set, we cannot rule out the presence of lower luminosity companions that are also able to drive a wind, particularly for the most luminous stars. A further suggestion for those stars that have left the main sequence is that they could have undergone phases - for example, as a red supergiant where a cooler photosphere may have driven a dusty wind which is now being cleared. If either of these scenarios is accurate, it would provide an efficient way for a stellar population to produce large quantities of dust before the appearance of red supergiants, supernovae, and AGB stars, which may help to explain the observations of anomalously high dust content of high-redshift galaxies (Omont et al. 2001). All of these scenarios would be expected to produce highly inhomogeneous dust distributions, allowing for identification with resolved high-sensitivity thermal/submillimetre imaging. A disturbed large-scale thermal structure appears in some of our sources and one example is provided by the Spitzer/MIPS $24 \mu \mathrm{m}$ image of HD 206773 shown in Fig. 6. Colliding wind binaries would produce a dense dusty region between the two stars, while the other two scenarios would produce clumpy circumstellar shells. Finally, we wish to stress the faintness of the dusty parsec-scaled halos, with a total optical depth as low as $\lesssim 10^{-4}$ and dust masses below that of Jupiter.

\subsection{Influence on ISM extinction or polarisation}

The Voshchinnikov sample of stars is frequently used to study dust in the diffuse ISM (Hincelin et al. 2011; Boyarchuk et al. 2016; Zhukovska et al. 2016; Shchekinov et al. 2017), Siebenmorgen et al. 2018). All of the stars lie on sightlines with visual extinction of less than $2.5 \mathrm{mag}$, colour excess of a few tenths of a magnitude (Table 1), and polarisation of a few percent, which is assumed to be of interstellar origin. The observed polarisation curves follow the Serkowski-law which is explained 


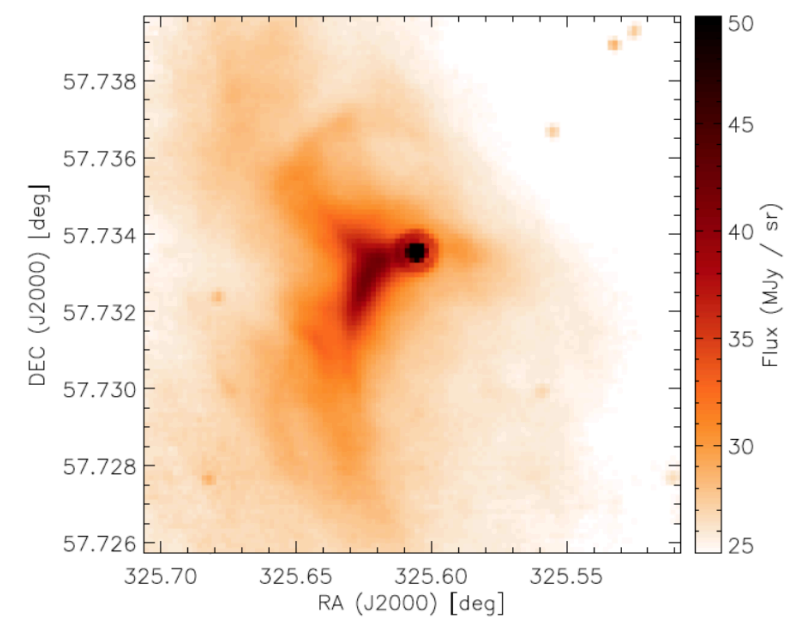

Fig. 6. Spitzer/MIPS $24 \mu \mathrm{m}$ image of HD 206773. Right ascension and declination is in degrees, the colour bar shows flux in $\mathrm{MJy} \mathrm{sr}^{-1}$. We note the large-scale background structure to the west.

by dichroic absorption by aligned dust grains and not by scattering on dust or free electrons (Voshchinnikov 2012). We are therefore able to confirm that for our subsample of 22 stars the polarisation originates in the ISM and not in the faint dusty halo around the stars. Even for the far-IR excess stars there is not sufficient circumstellar dust material available that could significantly contribute: their dust envelopes are too optically thin to influence the observed reddening. The shallow circumstellar dust halo also has no influence on the observed polarisation, whether caused by absorption or by scattering.

\section{Conclusion}

By comparing models with high-sensitivity Spitzer/IRS spectra we have found excess far-IR emission in $55 \%$ of a sample of 22 massive stars that are, with one exception, not known to be Be-stars and not connected to clouds. The excess becomes apparent at wavelengths $\gtrsim 10 \mu \mathrm{m}$ and is more pronounced in the far-IR. Black-body fits rule out the possibility of background source contribution to the excess. We successfully applied wind and dust models to account for the detected far-IR excess. Distinguishing or quantifying the contribution between these scenarios requires high-sensitivity resolved imaging observations at submillimetre wavelengths.

Spitzer/IRS offers the great advantage of observing mid-IR fine structure lines simultaneously with the far-IR continuum. This is important because of the transient nature of the excitation, as we demonstrate here. In stars with far-IR excess we detect Pf $\alpha$ in 10 out of 12 sources while for non-far-IR excess stars the detection rate drops down to $50 \% \operatorname{Pf} \alpha$ and $33 \%$ in $\mathrm{Hu} \alpha$, and where the line intensities become an order of magnitude weaker.

By means of echelle high-resolution spectroscopy we detect (or not) optical lines in $\mathrm{H} \alpha, \mathrm{C}$ III and N III irrespective of the presence of a far-IR excess. However, the detection rate of $\mathrm{H} \alpha$ is higher $(60 \%)$ for far-IR excess stars than it is for non-far-IR excess stars $(\sim 14 \%)$. We monitored HD 149757 between 1993 and 2010 and find weak $\mathrm{H} \alpha$ emission in only one spectrum taken in 2006. For the Be-star HD 47839 we do not detect $\mathrm{H} \alpha$ emission in a spectrum taken in 1996.

For far-IR excess, which is due to dust emission, the bulk material is located at parsec scales around the star in an extremely optically thin $\left(A_{\mathrm{V}, \mathrm{CS}}<500 \mu \mathrm{m}\right)$ circumstellar halo. The circumstellar dust halos are too faint to contribute to the observed extinction and dichroic polarisation observed along the sightline towards these sources. However, any significant dust population related to stars of such high luminosity may require continuous replenishment to be long-lived. Coronagraphic highcontrast imaging with SPHERE/IRDIS for three of these stars rules out the possibility that they host debris-disk-like populations of large rocky bodies whose collisions might provide a reservoir of material. Alternatively, the dust may be formed in situ in the wind or as a result of wind-wind interactions, possibly providing a channel for dust formation at high-redshift. The existence of such dust may be detectable in a $24 \mu \mathrm{m}$ Spitzer image.

Acknowledgements. We thank Robert Klement for discussions on Be-stars, T.D. Banas for supporting us in the SPHERE data reduction, and B. Altieri for help on the Herschel photometry. We are grateful to Nikolai Voshchinnikov for helpful comments and suggestions. This research is based on data obtained from the ESO Science Archive Facility and in particular on observations collected under ESO programme 095.C-0158(A). This research has made use of the SIMBAD database, operated at CDS, Strasbourg, France. This work is based in part on observations made with the Spitzer Space Telescope, which is operated by the Jet Propulsion Laboratory, California Institute of Technology under a contract with NASA. This research is based on observations with AKARI, a JAXA project with the participation of ESA. This publication makes use of data products from the Two Micron All Sky Survey, which is a joint project of the University of Massachusetts and the Infrared Processing and Analysis Center/California Institute of Technology, funded by the National Aeronautics and Space Administration and the National Science Foundation. This publication makes use of data products from the Wide-field Infrared Survey Explorer, which is a joint project of the University of California, Los Angeles, and the Jet Propulsion Laboratory/California Institute of Technology, funded by the National Aeronautics and Space Administration.

\section{References}

Bagnulo, S., Cox, N. J. L., Cikota, A., et al. 2017, A\&A, 608, A146 Banas, T. D. 2017, Master Thesis, Ludwig Maximilian University, München Barlow, M. J. 1979, in Mass Loss and Evolution of O-T Stars, eds. P. S. Conti \& C. W. H. De Loore, IAU Symp., 83, 119

Benaglia, P., Vink, J. S., Martí, J., et al. 2007, A\&A, 467, 1265

Beust, H. 2010, EAS Pub. Ser., 41, 219

Beuzit, J. L., Feldt, M., Dohlen, K., et al. 2008, Proc. SPIE, 7014, 701418

Bowen, D. L., Jenkins, E. B., Tripp, T. M., et al. 2008, ApJS, 176, 59

Boyarchuk, A. A., Shustov, B. M., Savanov, I. S., et al. 2016, Astron. Rep. 60, 1

Carciofi, A. C., \& Bjorkman, J. E. 2006, ApJ, 639, 1081

Castelli, F., \& Kurucz, R. L. 2003, IAU Symp., 210P, A20C

Castelli, F., \& Kurucz, R. L. 2004, ArXiv e-prints [arXiv:astro-ph/0405087]

Catanzaro, G. 2013, A\&A, 550, A79

Chojnowski, S. D., Whelan, D. G., Wisniewski, J. P., et al. 2015, Cat, 51490007C Cutri, R. M., Skrutskie, M. F., van Dyk, S., et al. 2003, VizieR Online Data Catalog: II/246

Cutri, R. M., Wright, E. L., Conrow, T., et al. 2012, VizieR Online Data Catalog: II/311

Draine, B. T. 2011, Physics of the Interstellar and Intergalactic Medium (Princeton: Princeton University Press)

Draper, Z. H., Wisniewski, J. P., Bjorkman, K. S., et al. 2014, ApJ, 786, 120

Ekström, S., Georgy, C., Eggenberger, P., et al. 2012, A\&A, 537, A146

Fabregat, J., Torrejon, J. M., Reig, P., et al. 1996, A\&AS, 119, 271

Fazio, G. G., Hora, J. L., Allen, L. E., et al. 2004, ApJS, 154, 10

Fitzpatrick, E. L. 2004, in Astrophysics of Dust, eds. A. N. Witt, G. C. Clayton, \& B. T. Draine, ASP Conf. Ser., 309, 33

Fitzpatrick, E. L., \& Massa, D. 2007, ApJ, 663, 320

Gaia Collaboration (Brown, A.G.A., et al.) 2018, A\&A, 616, A1

Galazutdinov, G. A., \& Krełowski, J. 2006, ApJ, 637, 342

Gordon, K. D., Cartledge, S., \& Clayton, G. C. 2009, ApJ, 705, 1320

Hall, J. S. 1958, Publications of the U.S. Naval Observatory Second Series, 17, 275

Hartmann, L., \& Cassinelli, J. P. 1977, ApJ, 215, 155

Heap, S. R., Lanz, T., \& Hubeny, I. 2006, ApJ, 638, 409

Hincelin, U., Wakelam, V., Hersant, F., et al. 2011, A\&A, 530A, 61

Houck, J. R., Roellig, T. L., van Cleve, J., et al. 2004, ApJS, 154, 18

Ishihara, D., Onaka, T., Kataza, H., et al. 2010, VizieR Online Data Catalog: II/297

Jenkins, E. B. 2009, ApJ, 700, 1299 
Joint Iras Science, W. G. 1994, VizieR Online Data Catalog: II/125

Kambe, E., Hirata, R., Ando, H., et al. 1997, ApJ, 481, 406

Klement, R., Carciofi, A. C., Rivinius, T., et al. 2017, A\&A, 601, A74

Kramida, A., Ralchenko, Y., Reader, J., and N. A. Team 2018, NIST Atomic Spectra Database (ver. 5.5.2). Available at https://physics.nist.gov/ asd (Accessed: 2018, January 25), National Institute of Standards and Technology, Gaithersburg, MD

Krełowski, J., Strobel, A., Vješnica, S., et al. 2018, MNRAS, 476, 4987

Krügel, E. 2008, An introduction to the physics of interstellar dust (Bristol: IoP)

Lafreniere, D., Marois, C., Doyon, R., et al. 2008, ApJ, 660, 770

Lamers, H. J. G. L. M., \& Leitherer, C. 1993, ApJ, 412, 771

Levenhagen, R. S., \& Leister, N. V. 2006, MNRAS, 371, 252

Lin, C.-C., Hou, J.-L., Chen, L., et al. 2015, yCatp, 040001504L

Markwardt, C. B. 2009, in Astronomical Data Analysis Software and Systems XVIII, eds. D. A. Bohlender, D. Durand, \& P. Dowler, ASP Conf. Ser., 411, 251

Martins, F., Schaerer, D., Hillier, D. J., et al. 2005, A\&A, 441, 735

Marton, G., Calzoletti, L. P., Garcia, A. M., et al. 2017, ArXiv e-prints [arXiv:1705.05693]

Mathis, J. S., Mezger, P. G., \& Panagia, N. 1983, A\&A, 128, 212

Megier, A., Strobel, A., Galazutdinov, G. A., \& Krełowski, J. 2009, A\&A, 507, 833

Neugebauer, G., Habing, H. J., van Duinen, R., et al. 1984, ApJ, 278, L1

Omont, A., Cox, P., Bertoldi, F., et al. 2001, A\&A, 374, 371

Puls, J., Markova, N., Scuderi, S., et al. 2006, A\&A, 454, 625

Rauw, G., Herve, A., Naze, Y., et al. 2015, A\&A, 580, A59

Rivinius, T., Stefl, S., \& Baade, D. 2006, A\&A, 459, 137

Rivinius, T., Carciofi, A. C., \& Martayan, C. 2013, A\&ARv, 21, 69

Scicluna, P., \& Siebenmorgen, R. 2015, A\&A, 584, A108
Scicluna, P., Siebenmorgen, R., \& Blommaert, J. 2017, IAU, 329, 1666 Shchekinov, Y. A., Lukash, V. N., Mikheeva, E. V., et al. 2017, PhyU 60, 961 S Siebenmorgen, R., Krügel, E., \& Mathis, J. S. 1992, A\&A, 266, 501 Siebenmorgen, R., Krügel, E., \& Laureijs, R. J. 2001, A\&A, 377, 735 Siebenmorgen, R., Voshchinnikov, N. V., \& Bagnulo, S. 2014, A\&A, 561, A82 Siebenmorgen, R., Voshchinnikov, N. V., Bagnulo, S., et al. 2018, A\&A, 611, A5 Skiff, B. A. 2013, yCat, 1, 2023

Skrutskie, M. F., Cutri, R. M., Stiening, R., et al. 2006, AJ, 131, 1163

Sloan, G. C., Kraemer, K. E., Price, S. D., \& Shipman, R. F. 2003, ApJS, 147, 379

Smith, B. A., \& Terrile, R. J. 1984, Science, 226, 1421

Sota, A. M., Apellániz, J., Walborn, N. R., et al. 2011, ApJS, 193, 24

Sota, A. M., Apellániz, J., Morrell, N. I., et al. 2014, ApJS, 211, 10

Su, K. Y. L., Rieke, G. H., Malhotra, R., et al. 2013, ApJ, 763, 118

Valencic, L. A., Clayton, G. C., \& Gordon, K. D. 2004, ApJ, 616, 912

van Loon, J. T., \& Oliveira, J. M. 2003, A\&A, 405, L33

Vieira, R. G., Carciofi, A. C., Bjorkman, J. E., et al. 2017, MNRAS, 464, 3071

Vink, J. S., de Koter, A., \& Lamers, H. J. G. L. M. 2001, A\&A, 369, 574

Vogt. S., \& Penrod, G. D. 1983, ApJ, 275, 661

Voshchinnikov, N. V. 2012, J. Quant. Spectr. Rad. Transf., 113, 2334

Voshchinnikov, N. V., \& Henning, T. 2010, A\&A, 517, A45

Voshchinnikov, N. V., Henning, T., Prokopjeva, M. S., \& Das, H. K. 2012, A\&A, 541, A52

Williams, P. M. 2008, Rev. Mex. Astron. Astrofis., 27, 71

Wisniewski, J. P., Bjorkman, K. S., Magalhaes, A. M., et al. 2007, ApJ, 671, 2040

Wright, C. M., D. Duy, T., \& Lawson, W. 2016, MNRAS, 457, 1593

Zhukovska, S., Dobbs, C., Jenkins, E. B., \& Klessen, R. S. 2016, ApJ, 831, 147

Zorec, J., \& Briot, D. 1997, A\&A, 318, 443 